\title{
COVID-19 and Cardiac Surgery: The perspective from the UK
}

\author{
Amer Harky ${ }^{1}$, Deborah Harrington ${ }^{1}$, Omar Nawaytou ${ }^{1}$, Ahmed Othman ${ }^{1}$, Catherine \\ Fowler $^{2}$, Gareth Owens ${ }^{2}$, Francesco Torella ${ }^{3}$, Manoj Kuduvalli ${ }^{1}$, and Mark Field ${ }^{1}$ \\ ${ }^{1}$ Liverpool Heart and Chest Hospital NHS Foundation Trust \\ ${ }^{2}$ Aortic dissection awareness UK and Ireland \\ ${ }^{3}$ University of Liverpool Faculty of Health and Life Sciences
}

September 2, 2020

\begin{abstract}
The emergence of Severe Acute Respiratory Syndrome Coronavirus 2 (SARS-CoV-2) in December 2019, presumed from the city of Wuhan, Hubei province in China and the subsequent declaration of the disease as a pandemic by the World Health Organization (WHO) as COVID-19 in March 2020, had significant impact on health care systems globally. Each country responded to this disease in different ways but broadly by fortifying and prioritising health care provision as well as introducing social lockdown aiming to contain the infection and minimizing the risk of transmission. In the United Kingdom, a lockdown was introduced by the government on 23rd of March 2020 and all health care services were focussed to challenge the impact of COVID-19. To do so, the United Kingdom National Health Service had to undergo widespread service reconfigurations and the so-called "Nightingale Hospitals" were created de novo to bolster bed provision and industries were asked to direct efforts to the production of ventilators. A government led public health campaign was publicised under the slogan of: "Stay home, Protect the NHS (National Health Service), Save lives". The approach had a significant impact on delivery of all surgical services but particularly cardiac surgery with its inherent critical care bed capacity. This paper describes the impact on provision for elective and emergency cardiac surgery in the United Kingdom, with a focus on Aorto-vascular disease. We describe our Aorto-vascular activity and outcomes during the period of UK lockdown and present a patient survey of attitudes to aortic surgery during COVID-19 pandemic.
\end{abstract}

\section{Background to COVID-19 and effects on services for Cardiac Surgery in UK}

The Severe Acute Respiratory Syndrome Coronavirus 2 (SARS-CoV-2) first emerged in the city of Wuhan, China, in December 2019 and it has since spread rapidly across the globe, causing a disease named as COVID19 in February 2020 and with the World Health Organisation (WHO) declaring a pandemic in March 2020 (1). As of $20^{\text {th }}$ of August 2020, there are more than 22.2 million confirmed cases of COVID-19 and over 795,000 deaths reported globally (Figure 1) with the United Kingdom (UK) being $12^{\text {th }}$ among countries in term of confirmed cases (more than 326,000) and $5^{\text {th }}$ in COVID-19 related deaths (more than 41,000, Figure $2),(2)$.

Public Health England (PHE) published the very first report on COVID-19 on 22 ${ }^{\text {nd }}$ January 2020. Just one day later, the Emergency Department at Royal London Hospital swabbed its first potential COVID-19 patient (3). The declaration of this disease as a pandemic put health care systems in the UK on alert and the government introduced national lockdown on $23^{\text {rd }}$ of March 2020 in an attempt to contain the disease and minimize the transmission risk to others. A campaign was launched under the slogan of "Stay home, Protect the NHS (National Health Service) and Save lives". Although a critical step to combat this highly contagious disease, it created a significant burden on an otherwise a freely accessibly health care system, the NHS. The NHS had to undergo a significant transformation diverting resources to frontline health care services including ambulance services, emergency departments and allocation of intensive care beds 
in preparation for the potential influx of COVID-19 patients and the requirement for ventilatory support (Figure 3). De novo facilities, the Nightingale Hospitals, were created throughout the nation to increase capacity; private hospital capacity was purchased, industries were tasked with producing ventilators and academia with producing treatments and vaccines. Effectively, all elective care was stopped with services only maintained for emergencies.

Amongst the many specialities affected by the NHS service reconfiguration was cardiac surgery, given its ownership of a large resource of ventilated beds normally required in elective practice. Attempts were made in a number of regions to create centralized cardiac surgical services to continue provision of care to this high-risk cohort and avoid secondary deaths due to untreated cardiovascular diseases (4). The Royal College of Surgeons (RCS), Society of Cardiothoracic Surgery in UK and Ireland (SCTS) and the NHS issued guidelines and regular updates on the practice of cardiac surgery during this pandemic, introducing protocols and pathways to minimize the risk of COVID-19 to patients and staff without affecting the quality of service and care to those needing cardiac surgery (5-11).

The network of centres that perform cardiac surgery in England generally responded to the crisis according to government guidance by reducing or, more frequently, halting elective operating, but with a degree of independence. The exact timeline during which each centre wound down elective and urgent services varied according to local circumstances and pressures. The processes by which each centre managed patient pathways were dependent on local arrangements. In addition, England, Wales, Scotland and Northern Ireland, each with its own devolved government, responded differently. This paper focusses on the experience of Liverpool Heart and Chest Hospital with changes to service provision for cardiac surgery, focussing on aorto-vascular patients.

\section{Recommendations and Guidelines during COVID-19 in Britain}

On $20^{\text {th }}$ March 2020, the Royal college of Surgeons (RCS) published its initial, brief guidance for surgeons who were working during the COVID-19 pandemic, emphasizing the safety of the working force as well as the maintenance of emergency surgical workforce and capabilities (5). The detailed guidance came into force on $26^{\text {th }}$ of March 2020 outlining the scope of patient selection and flow of surgical practice across the UK. Since then, the guidelines have been updated four times, lastly on $5^{\text {th }}$ June 2020.

The initial guidance involved cancellation of all elective operating cases, with focus on operating on urgent/emergency and otherwise life-saving procedures (6). Patients were categorised into four levels according to their need for surgery:

- Priority level 1a Emergency - operation needed within 24 hours

- Priority level $1 \mathrm{~b}$ Urgent - operation needed with 72 hours

- Priority level 2 Surgery that can be deferred for up to 4 weeks

- Priority level 3 Surgery that can be delayed for up to 3 months

- Priority level 4 Surgery that can be delayed for more than 3 months

With the gradual decline in the cases of COVID-19, the service gradually resumed its activities, slowly reintroducing elective surgery on a phased basis. Elective cases were prioritised as Red, Amber, Green (RAG rating) with red been classified as "urgent elective".

With a similar approach but at a more specialized level, the SCTS introduced national guidelines on the performance of cardiac surgery. As its initial response, the society introduced a clear cardiothoracic surgery escalation framework on $16^{\text {th }}$ and $18^{\text {th }}$ March 2020; which outlined the routine practice of operating theatres, clinics and the running of multi-disciplinary team (MDT) meetings (7). It classified cardiothoracic patients in 4 areas, the obligatory in-patients, which required surgical intervention, the alternative (non-surgical) pathways including inpatients and those to be managed by ambulatory base services, the day-cases and, finally, the outpatients, whose hospital visits were to be kept at the minimum safe level. The society also developed a clear pathway for patient selection during the initial lockdown and to smooth the gradual resumption of elective activity. The guidelines not only included patient selection but also focused on triage 
methods of such cohort, COVID-19 screening methods and tests, the use of PPE and the management of operating theatres. These guidelines were implemented nationwide and helped in containing the spread of COVID-19 in cardiac surgery patients. (8) The society's latest guideline on resumption of elective activity eliminates the requirement for pre-operative radiological screening if they have been self-isolating for 14 days prior to surgery, provided that they have no COVID-19 related symptoms and have negative COVID-19 nasopharyngeal swab within 72 hours of surgery date. (9)

The NHS also issued several, nationwide guidelines to provide insights on speciality practice during COVID19 pandemic. Most of the clinical guidelines and recommendations were interlinked with the work of the RCS and SCTS. The NHS and PHE recognized that cardiothoracic surgery, like any other speciality, needed service modification which depended on the unit and the region of service, considering that some cardiothoracic units are incorporated as part of a large trauma centres while others are tertiary units without emergency department service (10). The NHS categorized the patients into 6 major groups:

1. Obligatory in-patients : Those patients who need immediate admission and surgical intervention

2. Alternative pathways : this is categorized into two subgroups:

a. In-patient: the condition can reasonably be managed on an ambulatory basis after a more limited inpatient stay than normal; eg ambulatory chest drain management.

b. Ambulatory: the condition can reasonably be managed on an ambulatory basis.

3. Day-cases : Surgery can be safely undertaken for a large number of conditions.

\section{Surgery and interventional care that can be postponed}

\section{Trauma surgery.}

\section{First contact and clinics.}

In addition to above, the work of the cardiothoracic team was expanded to have a consultant led service, including patient assessment, daily reviews and decision-making process. The NHS also advised to restructure training and education needs during this time period to give priority to COVID-19 patients care provision $(12,13)$. In its latest guide, the NHS advised to utilize a remote consultation, where appropriate. However, when face-to-face consultations were needed, patients were brought in for further assessment in a controlled and organized manner (11).

PHE, NHS, SCTS and RCS eventually merged their statements to restructure the daily practice of cardiac surgery including modification of hospital setups, patient selection and screening process as well as standards for intubation, operating and provision of perioperative care for such patients. The joint statements were released in accordance to the severity of COVID-19 pandemic within the UK general population and the phase of the disease.

\section{Service transformation}

The NHS has been stretched to provide care for the already aging population alongside the new cases of infected COVID-19. As such and due to limited capacity, there have been some attempts at reconfiguration of services, in some regions, by creating centralized units to provide care for sub-specialities that are not in direct response to COVID-19. This service modification entailed the creation of detailed and tailor-made protocols for planning cardiac surgery whilst optimising the use of intensive care and ward beds for the treatment of COVID-19 cases. Such process required nationwide assessment of capacity and capabilities to accommodate such changes. In the North-West of England, which serves a population of 7.3 million, cardiac care was channelled through four major cardiothoracic units: Blackpool, Manchester Royal, Manchester Wythenshawe and Liverpool Heart and Chest Hospital (LHCH); LHCH was chosen to be the central unit for cardiac and aortic surgery and led the development of the North-West Urgent Cardiothoracic Service (NUCS) Protocol to guide patient treatment pathways (Appendix 1). As NUCS was set up, government measures took effect, reducing admissions; in reality few patients were channelled into Liverpool from other 
cardiac units, but some throughput continued from our usual catchment area. North-West regional pathways still exist in preparation for a potential second spike. Similarly, in London the service was reconfigured to operate in only two units among the combined 7 NHS centres serving the population of 8.5 million people, forming the Pan London Emergency Cardiac Surgery (PLECS) service (14). It is important to emphasize that the base of developing such centralized services and detailed protocol was to provide a COVID-19 free environment for patients undergoing cardiac surgeries. This is a very critical point as COVID-19 seems to have significant correlation with cardiovascular diseases and outcomes (15-17).

Maintenance of a COVID-19 free environment with clean patient pathways was key to maintaining a limited but safe service. There was significant reduction in the operational activities, as high as $83 \%$ in some cardiac surgical units (4). Our centre observed similar reductions (Figure 4). Eventually, the establishment of standardised patient pathways (Appendix 2) for perioperative care and management in theatre (Appendix 3) aided in a gradual increase in the surgical activities. According to regional pathways (NUCS and PLECS), patients were classified into four major categories:

- Level 1: Elective patients who have indications for routine cardiac surgery and would normally be added to an elective waiting list.

- Level 2: Urgent patients at home who are on the existing waiting lists or in the process of referral but have critical / life threatening anatomy with worsening symptoms or the need for urgent prognostic intervention.

- Level 3: Urgent patient undergoing inter-hospital transfers who by definition are in hospital with prognostic / critical anatomy or physiology or with unstable symptoms. They require cardiac surgery within this hospital admission (but not on the same day), and no other options for treatment are possible such as medical or percutaneous intervention.

- Level 4: Emergency cases which are most commonly acute aortic dissections, such patients have life threatening emergency aortic and cardiac conditions and require surgery within hours.

For NUCS the decision-making process started with the receipt of an urgent inpatient referrals, after triage at the referring regional cardiac hospital (Blackpool and Manchester). These were directed to our local COVID-19 daily multidisciplinary team meeting (MDT) along with our local urgent referrals. All our 10 weekly MDTs were amalgamated into a single and virtual COVID-19 MDT with widespread attendance. After review of the available information, an outcome was communicated to the referring clinical team and the patient. If intervention was deemed necessary, then procedural planning took place and the case was allocated to a consultant and date for surgery identified. Emergency referrals were processed in the usual way by on-call staff. A number of patients requiring emergency care were referred to Liverpool on the basis on the NUCS arrangement.

\section{Aorto-vascular disease and COVID-19 at Liverpool Heart and Chest Hospital}

Liverpool Heart and Chest Hospital (LHCH) is one of a very few centres in the UK offering medical and surgical services for patients with complex aorto-vascular diseases. The hospital is the only stand-alone Trust in the UK offering only cardiovascular and thoracic services and as such has no Emergency Department or Acute Medical Admissions facility. Four of fifteen cardiac surgeons specialise in aortovascular surgery with a separate emergency on-call rota. The team also work with local vascular surgeons under the banner of Liverpool Cardiovascular Surgery (LCS), with regular joint operating, commonly on hybrid cases. From $23^{\text {rd }}$ March onward, the independent elective listing of patients for surgery by consultants was abandoned. General cardiac activity was wound down, under the direction of central government, to free up critical care capacity for potential transfer of COVID-19 patients from acute hospitals in the region. Activity was reduced from 5 cardiac theatres and 10 cases per day to 4 theatres and 4 cases per day, with only urgent patients allocated from a common pool. Aorto-vascular patients, urgent and emergency, had to compete with cardiac surgical patients for theatre space. All patients were discussed at t daily virtual "COVID-19 MDT" where an emphasis was placed on directing patients towards medical or minimally invasive therapy (endovascular) whenever possible. With time, a number of high-risk elective patients were operated. 


\section{Risk assessment of elective aortovascular patients}

A major issue in this period was the quantification of post-operative COVID-19 infection in "clean" patients, thus balancing the additional risks of death from viral infection versus the risk of a putative delay in surgery - a delay of at least three months was presumed. For aorto-vascular disease the Vascular Society of Great Britain \& Ireland (UK) offered guidance by increasing the size threshold for elective intervention for abdominal aortic aneurysm (AAA) to $>7 \mathrm{~cm}$ (18) as did the Society for Vascular Surgery in the United States, recommending intervention only on symptomatic thoraco-abdominal disease (19). The evidence base underlying this advice was opaque at best. We "RAG rated "(Red, Amber Green) and chose to operate on the so-called "Red urgent elective" patients with COVID screening and "clean hospital pathways". The definition of Red was symptomatic severe disease. During this period, we made no adjustments to size-based guidelines.

\section{Emergency aorto-vascular patients}

There were unanimous recommendations from all advisory groups to treat emergency life threatening disease as normal while adopting appropriate safeguarding procedures for staff and other patients within the hospital.

\section{Referral activity}

A commonly observed phenomenon during this period was a dramatic reduction in both elective and urgent/emergency referrals thought to be due to very few patients presenting to hospital due to a fear of COVID-19 and local triage by referring doctors.

\section{Outcomes of operated aorto-vascular patients}

We examined our outcomes between the dates of $1 / 3 / 2020$ and $4 / 7 / 2020$. A total of 59 patients were operated (Table 1) during this period. In normal times we would expect the 4 aortovascular surgeons to perform roughly 1 elective/urgent case each per week over 42 weeks per year (i.e. total 56 cases) plus emergencies, suggesting our aortovascular activity was largely maintained during this 14-week period.

1. Elective (Red on RAG rated)During this period, we performed operations on elective patients including root, arch, descending thoracic aorta (DTA) and thoraco-abdominal aortic aneurysm (TAAA) surgery including thoracic endovascular aortic repair (TEVAR). One of these elective patients turned COVID19 positive in the post-operative period but did not develop COVID-19 pneumonia; the COVID-19 related mortality was zero.

2. UrgentUrgent patients were those referred in from other hospitals and in-house patients requiring surgery during the same admission. Patients were screened for COVID-19 at referring hospitals and underwent CT screening and repeat COVID-19 swabs, lactic dehydrogenase (LDH) assay and lymphocyte measurements on transfer. We operated on 21 such patients. None developed COVID-19 but there were 3 deaths.

3. EmergencyEmergency patients came into our unit from referring hospitals and were taken to theatre immediately with COVID-19 status unknown. We operated on nine such patients, two of whom developed COVID-19 in the post-operative course but not COVID-19 pneumonia. There was one non-COVID-19-related death.

4. Medically managed patientsWe managed 15 aorto-vascular patients without surgery either because it was not indicated or because patients were unfit for the necessary surgical procedure. Eight were Type A dissections (moribund, 3; major stroke, 1; sub-acute, 1; or patient too frail/comorbid; 3). Five patients had surgically relevant thoraco-abdominal aortic dissection or aneurysm but were too frail/comorbid; one was an uncomplicated acute Type B (COVID-19 positive). One patient had a root abscess that was COVID positive and died while awaiting a negative swab prior to transfer.

No patient in this cohort died of post-operative COVID-19 pneumonia. It should be noted that our critical care area is divided into 4 distinct rooms, an arrangement that facilitated isolation of COVID-19 positive patients. During this period, we regularly admitted ventilated patients from neighbouring acute hospitals with community-acquired COVID-19. In summary, we attempted to maintain our aortovascular patients 
COVID-19-free via a combination of preoperative screening, strict theatre procedures, and separate pathways the "clean" and the COVID-19 cohort (Appendices 1-3).

It should be noted that our preoperative screening protocols changed as evidence presented itself. At the start of the lockdown period we performed routine CT scanning and bronchalveolar lavage (BAL) in theatre or when a patient returned to ITU. During late June 2020, we eventually abandoned CT scanning and a plain chest radiograph was used instead to identify individuals with early or suspected COVID pneumonia. In addition, it became clear the BAL was highly sensitive in the detection of viral RNA, but it was unclear whether this was simply dead virus indicating previous exposure or rather an active infection. Our experience showed that a positive BAL was of no consequence for the clinical course of the patient but created major issues for bed capacity with a need for isolation. For this reason and during late July, BAL was stopped in elective patients with a pre-operatively negative COVID-19 swab, normal chest x-ray and blood tests who had been isolating for two weeks.

We are thus only aware of one patient who should have undergone urgent surgery for a root abscess but died following delays, while awaiting his status to change from COVID-19 positive to negative. To our knowledge, no patients came to harm while on our waiting lists for delayed elective surgery. We see this as validation of the systems we developed to balance the need to make our critical care beds available for the national COVID-19 pandemic and the needs of our patients with life-threatening cardiovascular disease.

After this period, we gradually returned to normal work patterns, with surgeons planning their operating lists independently, progressively increasing elective activity as hospital pathways allowed. We still use a RAG rating system at present.

\section{Patient perspective}

In order to better understand the beliefs and attitudes of patients with aortic pathology during COVID-19, we conducted a survey through Aortic Dissection Awareness UK and Ireland (ADA UKI).

A structured questionnaire was developed and pre-operative patients who are members of ADA UKI were invited complete it from $17^{\text {th }}$ August and $25^{\text {th }}$ August 2020. A total of 29 responses were received and the results are presented in Table 2.

Among the 29 patients, only one of them considered himself as "awaiting surgery" while the rest 28 patients considered themselves as "under surveillance" although they have been offered the option of surgical intervention at time of first assessment and rather awaiting a confirmed date for surgery which has been significantly affected by COVID-19 pandemic.

More than $80 \%$ of them were more concerned about the delayed aortic surgery than the possibility of contracting COVID-19 in-hospital; over $70 \%$ of them had no concerns in attending hospital and trusted their respective unit to have strict measures in place to prevent cross-infection. They would have preferred to have surgery without delay despite the potential risk of COVID-19 (72\%). Furthermore, most would have preferred a face-to-face follow-up (59\%) while a clear majority did not feel that that a routine follow-up scan should be delayed pending vaccine development $(90 \%)$.

Our survey shows that, despite the potential risk of COVID-19, patients are more worried about their health from the underlying aortic pathology than the possibility of contracting COVID-19. As this was a simple cross-sectional survey on a small sample, results should be interpreted carefully; larger qualitative studies are needed to understand the impact of COVID-19 pandemic and its associated delays over patients who are yet to have aorto-vascular surgery.

\section{Outpatients}

To minimize the risk of transmission of COVID-19 to our patients and health care professionals, all elective face-to-face out-patient reviews were cancelled and turned into a virtual consultation by telephone or videolink. During this COVID-19 pandemic, telemedicine has been explored and utilized in many other specialities 
and it has proven its value in such prospect (20-22). With the passage of the peak of COVID-19, "face to face" consultations were re-established for a proportion of patients, with all necessary preventative measures.

\section{Restarting Services}

Pathways and standard operating procedures have evolved through multiple iterations in an attempt to return to normal pre-COVID activity levels while maintaining patient and staff safety. With the advent of effective separation of preoperative and post-operative patients, rapid COVID swabbing, radiological screening and preoperative patient shielding, we have approached $80 \%$ theatre operating capacity. Staff safeguarding is maintained through mandatory social distancing, virtual MDTs, face masks as well as track and trace methods. Today, most of the cardiac surgery centres across the UK have resumed their activity in a phased return, initially with urgent/emergency cases. Now, elective cases are considered for surgery on the basis of RCS guidelines and patient self-isolation for 14 days prior to admission.

\section{The New Normal and Summary}

Without doubt, the COVID-19 pandemic has caused significant disruption to services globally. One of the most affected sectors was healthcare provision, which required extensive support from governments and volunteers in order for it to provide safe care. Globally there has been service reconfiguration, with some units shutting down some of their services to cope with COVID-19 patient influx while others diverted their activity into a more centralized, regional hub to be able to deliver emergency, specialist services. NHS England has been in the frontline combating COVID-19 and cardiothoracic surgical services have been modified to reflect this. In England, the only regions with a clear cardiothoracic surgical pathway for the COVID-19 pandemic were London through PLEC and the North West of England.

Following service modifications, there remain thousands of patients affected by cancellation of their operation, clinic assessment or follow-up. The outcomes of this cohort is unknown; it will be of great interest to understand how these patients, and their quality of life, have been affected.

There is uncertainty about when a full cardiothoracic service will re-run and whether preoperative testing for COVID-19 will be a permanent requirement; data are emerging on daily base and to-date there are more than 47,000 entries in PubMed.gov related to COVID-19, increasing on daily base. Will the present state of affairs be the new norm for cardiac surgeons for the foreseeable future? How will the NHS provide its services in the future? Time and further research will address these questions.

ConclusionsThe COVID-19 pandemic has had significant impact on our nation causing death, disability, and resulting in incalculable effects on families, social structures through long-lasting consequences for our economy. Through changes in social behaviour, building of our bed base and changes in NHS structures and priorities, the country stopped the pandemic overwhelming our critical care capacity. There was, however, a significant impact on NHS health care provision including cardiac surgery. The burden of cardiovascular disease on morbidity and mortality as a consequence of these arrangements remain unknown at present. Our patient survey showed that patients are more worried about risks to their health from underlying aortic pathology than contracting COVID-19 in hospital and its associated perioperative risks. Cardiac surgeons have learnt an enormous amount on how to manage the service in the context of a national pandemic. Hopefully, this manuscript will offer some insight on how we managed the challenge.AcknowledgementsWe would like to acknowledge that many of the systems and processes described for LHCH are the result of input from multiple disciplines and individuals across different specialities including the management team. We are thankful to the team at Department of Anaesthesia and intensive care at LHCH for their efforts and inputs into the design of theatre protocols and COVID-19 Care Pathways at our hospital. We would also like to acknowledge that many of the pathways are edited versions from documents that are freely available and accessible on SCTS and RCS websites including PLECS, and are modified for local use. We are grateful to Professor Aung Oo at St. Bartholomew's Hospital, Barts Heart Centre, London, United Kingdom for sharing the early experience of COVID-19 in London and their development of Care Pathways. We would like to thank the patients of Aortic Dissection Awareness in contributing to the patient survey.Human Studies: No ethical approval required for this study as no patient identifiable information in included.References: 
1. World Health Organization. Naming the coronavirus disease (COVID-19) and the virus that causes it. 2020. https://www.who.int/emergencies/diseases/novel-coronavirus-2019/technical-guidance/namingthe-coronavirus-disease-(covid-2019)-and-the-virus-that-causes-it. [Accessed 22nd August 2020]

2. Dong E, Du H, Gardner L. An interactive web-based dashboard to track COVID-19 in real time [published correction appears in Lancet Infect Dis. 2020 Jun 12:]. Lancet Infect Dis. 2020;20(5):533534. doi:10.1016/S1473-3099(20)30120-1.

3. Avery, Joanna; Bloom, Benjamin COVID-19, a UK perspective, European Journal of Emergency Medicine: June 2020 - Volume 27 - Issue 3 - p 156-157 doi: 10.1097/MEJ.0000000000000700.

4. Mohamed Abdel Shafi A, Hewage S et al. The impact of COVID-19 on the provision of cardiac surgical services. J Card Surg. 2020;35(6):1295-1297. doi:10.1111/jocs.14631.

5. https://www.rcseng.ac.uk/coronavirus/joint-guidance-for-surgeons-v1/ accessed 22nd August 2020.

6. https://www.rcseng.ac.uk/coronavirus/surgical-prioritisation-guidance/. accessed 22nd August 2020

7. https://www.rcseng.ac.uk/coronavirus/recovery-of-surgical-services/. accessed 22nd August 2020

8. https://scts.org/covid-19/ accessed 22nd August 2020

9. https://scts.org/wp-content/uploads/2020/05/SCTS-Current-Recommendations-Regarding-PreOperative-COVID-19-Screening-CT-Scan-in-Patients-Undergoing-Cardiothoracic-Surgery-19th-May2020.pdf. accessed 22nd August 2020

10. https://www.england.nhs.uk/coronavirus/wp-content/uploads/sites/52/2020/03/specialty-guidecardiothoracic-surgery-v1-20-march-2020.pdf. accessed 22nd August 2020

11. https://www.england.nhs.uk/coronavirus/wp-content/uploads/sites/52/2020/03/C0044-SpecialtyGuide-Virtual-Working-and-Coronavirus-27-March-20.pdf. accessed 22nd August 2020

12. Shafi AMA, Atieh AE, Harky A, Sheikh AM, Awad WI. Impact of COVID-19 on cardiac surgical training: Our experience in the United Kingdom. J Card Surg. 2020;35(8):1954-1957. doi:10.1111/jocs.14693

13. Harky A, Chen R, Pullan M. Examining the impact of COVID-19 on cardiac surgery services: The lessons learned from this pandemic [published online ahead of print, 2020 Jul 9]. J Card Surg. 2020;10.1111/jocs.14783. doi:10.1111/jocs.14783

14. Hussain A, Balmforth D, Yates M, et al. The Pan London Emergency Cardiac Surgery service: Coordinating a response to the COVID?19 pandemic. J Card Surg. 2020;1-7. https://doi.org/10.1111/jocs.14747.

15. Zaim S, Chong JH, Sankaranarayanan V, et al.. COVID-19 and Multiorgan Response. Curr Probl Cardiol. 2020;45(8):100618. doi:10.1016/j.cpcardiol.2020.100618.

16. Khan IH, Zahra SA, et al. At the heart of COVID-19. J Card Surg. 2020;35(6):1287-1294. doi:10.1111/jocs.14596.

17. Shafi AMA, Shaikh SA, Shirke MM, Iddawela S, Harky A. Cardiac manifestations in COVID-19 patients-A systematic review. J Card Surg. 2020;35(8):1988-2008. doi:10.1111/jocs.14808.

18. https://www.vascularsociety.org.uk/_userfiles/pages/files/Newsletters/2020/Presidents\%20update\%2027_03_20.pdf. accessed 22nd August 2020

19. https://www.facs.org/covid-19/clinical-guidance/elective-case/vascular-surgery. accessed 22nd August 2020

20. Hollander JE, Carr BG. Virtually Perfect? Telemedicine for Covid-19. N Engl J Med. 2020;382(18):1679-1681. doi:10.1056/NEJMp2003539.

21. Leung MST, Lin SG, Chow J, et al. COVID-19 and Oncology: Service transformation during pandemic [published online ahead of print, 2020 Aug 18]. Cancer Med. 2020;10.1002/cam4.3384. doi:10.1002/cam4.3384

22. Shirke MM, Shaikh SA et al. Tele-oncology in the COVID-19 Era: The Way Forward?. Trends Cancer. 2020;6(7):547-549. doi:10.1016/j.trecan.2020.05.013

Appendices:These are local amendments to freely accessible documents published on the SCTS website (https://scts.org/covid-19/)

1. North-West Urgent Cardiothoracic Service 
2. LHCH Patient Pathway

3. LHCH Operative standard operating policy

Figure Legends:Figure 1. World Health Organization statistics of COVID-19 globally.Source www. WHO.int (accessed 22 $2^{\text {nd }}$ August 2020)Figure 2. United Kingdom COVID-19 status of confirmed cases and deaths.Source www.gov.uk (accessed 222 $2^{\text {nd }}$ August 2020)Figure 3. Status of patient admission to hospital and requirement of mechanical ventilation in COVID-19 patients in the United Kingdom.Source www.gov.uk (accessed 22 ${ }^{\text {nd }}$ August 2020)Figure 4. Average weekly Cardiac surgery activities at Liverpool Heart and Chest Hospital

\section{Hosted file}

Tables.docx available at https://authorea.com/users/312194/articles/478410-covid-19-andcardiac-surgery-the-perspective-from-the-uk
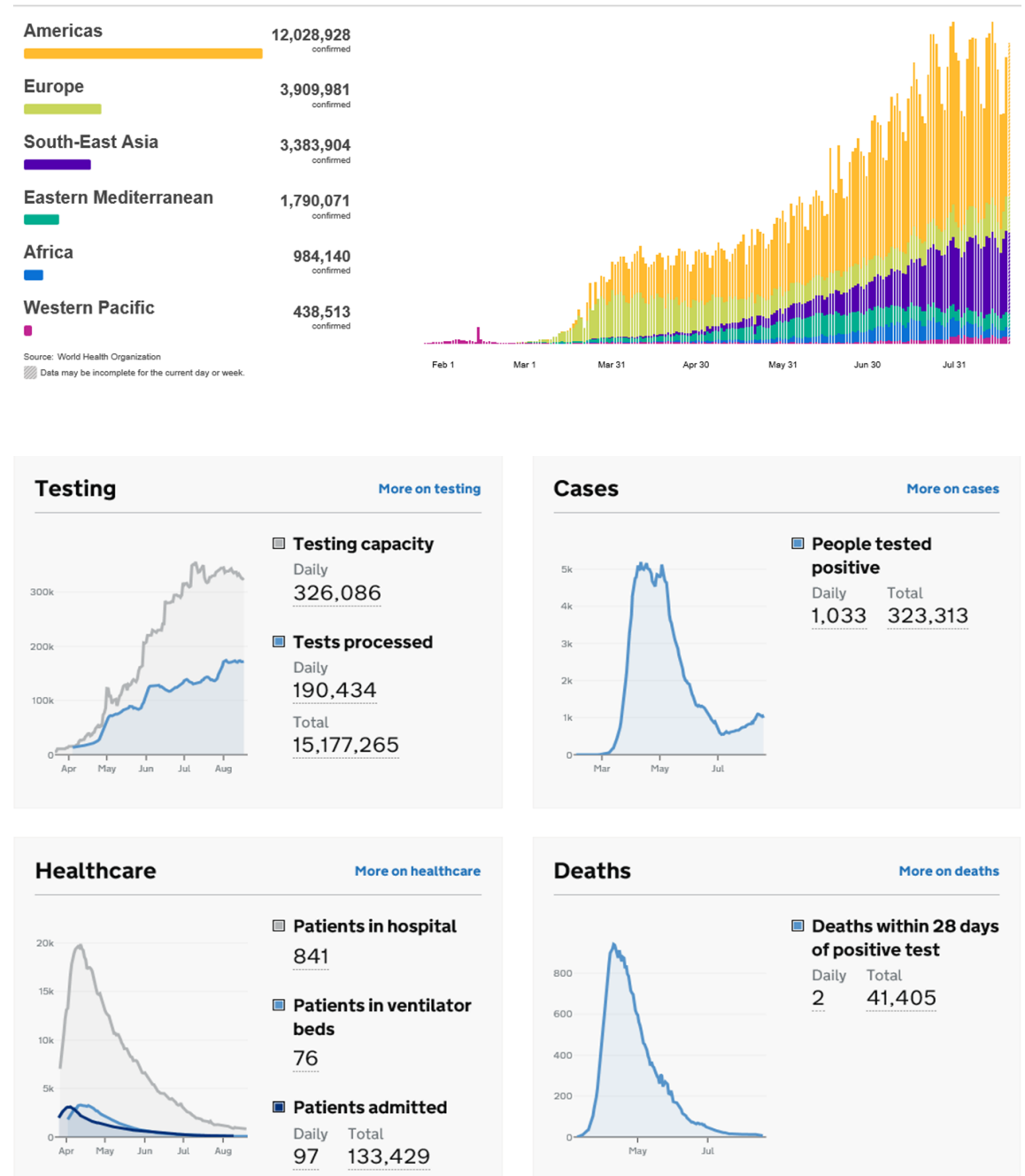
Patients admitted

Daily Total

$97 \quad 133,429$
Patients in hospital

841

Patients on ventilation

76

\section{Patients admitted to hospital}

Daily Cumulative Data About

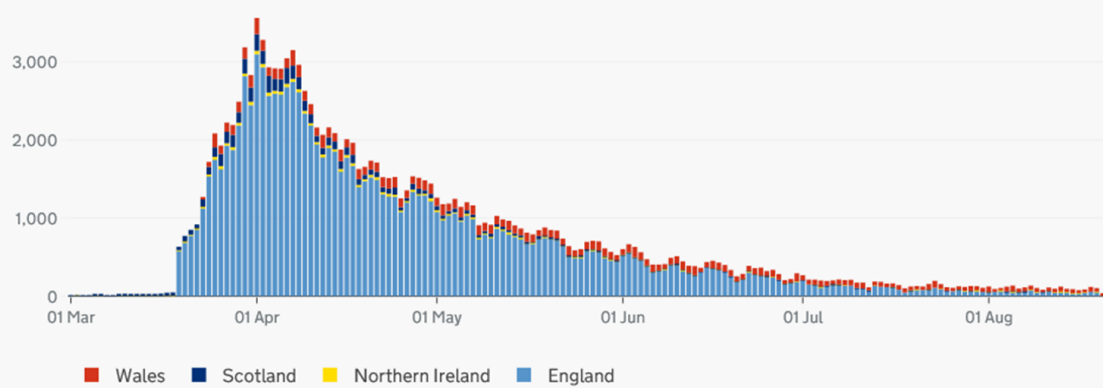

\section{Average weekly case}

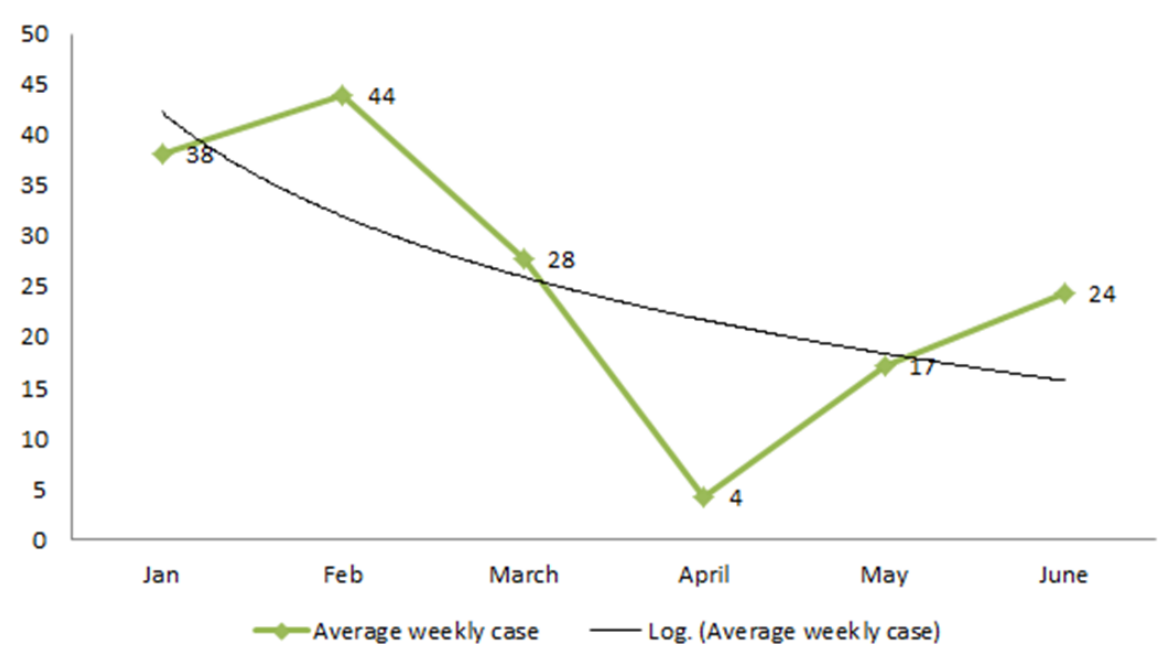

